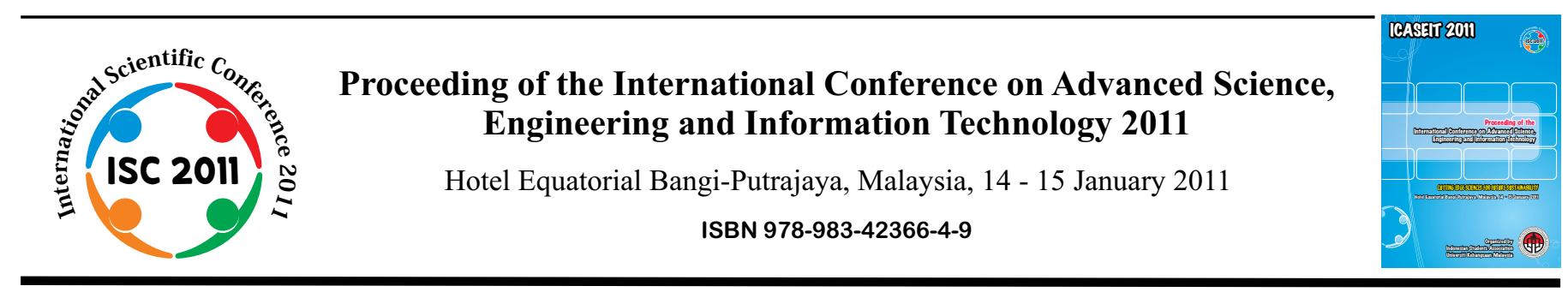

\title{
Logo Detection Using Pose Clustering and Momentums
}

\author{
Mohammad Hadi Karimi Tafti, ShahramRajaee, Hossein Sadeghi, Sadegh Tabatabaeifar \\ Department of Electronic Engineering Islamic Azad University, Mehriz branch \\ Mehriz, 8981883114, Iran \\ Tel.:+983525229100, E-mail: eng.m.h.karimi@gmail.com
}

\begin{abstract}
- nowadays, logo and arm detection with growing variety and number of arms in companies and countries is one of the significant topics in image processing.For logo detection there are many image processing algorithms which can be used for this purpose regard to features of the logo .In the most of recent works, images are exactly the logos, but in general, the logo can be one portion of another image or even can transform (rotate, skew, shift, ...) and have more complexity .In this paper we will select Iran logo as a sample, because it has not any regular geometric form and has special complexity .In a bank of images we will try to find this logo and its position with its transformation .Images bank includes images that has not Iran logo or has Iran logo with affine transform within another pictures .Approaches that will be discussed here are pose clustering and momentum clustering .Simulation results show that this approach can be used as a suitable way for finding the existence and position of arm in this field.
\end{abstract}

Keywords — Logo Detection, Pose Clustering, Momentum, Image Processing, Corner Detection.

\section{INTRODUCTION}

Recently, using of logo and arm detection due to its usage in companies and international societies is spreading and has been one of the important topics in image processing field . For logo detection, there are a lot of image processing algorithms that can be applied, but in the most of them, logos are in separate images in a regular format.But for advanced or commercial uses, arm or logo can be one part of a complex picture and even can be the transformed form (rotated, skewed, shifted ...) of it. Also, the problem of logo recognition has received a great deal of attention, primarily as an effective way to index or cluster documents such as letters or memos based on the identity of the originating institution. The fact that logos tend to be unique to a given institution, and that they remain relatively stable over long periods of time, makes them one of the few graphic components that can be indexed in this way [1].

In this paper we choose Iran logo as our logo, because it has enough complexity and does not have any specific geometric shape .Here we use a bank of images consists of two group, one group are negative group which consists of images that do not have our logo but have similar arms and logos, and the other group that consists of images that have our logo in them in a transformed shape which is placed in an unknown position of images .If it can be successful, it can easily be used in simpler logos.

Prior works related to logos have focused almost exclusively on logo recognition [2], [3], [4], [5], [7].Given a detected and precisely extracted logo, logo recognition attempts to classify the logo as belonging to one of the finite number of logo classes or conclude that it does not belong to any class .Most studies on logo recognition assume that an effective logo detection and extraction approach is available. Zhu in [6] uses SVM (Support Vector Machine ) to do that. Recently also some types of video logo detection are advancing [8], [9] .

In logo detection, at first we should choose special features for it and using that features find our logo among the pictures .First feature that looks like a good candidate for it is using edges as feature points .But edges are suitable chooses if our arm consists of simple lines .In this case we can use the ways such as hough transform [10] to extract features .But in a complex logo like Iran logo due to its shape and possible transformations, it is not a good candidate. Then we should find better candidate that can work in more complicated cases .This candidate should be 
able to detect affine transformation .Among the algorithms, pose clustering algorithm looks fine . This algorithm search for affine transform between every two interest point $\mathrm{s}$ (feature points) of training picture and test pictures . The best feature points for this aim look like to be using of corners which is better candidate for our approach. We use these points as our feature points in this article.

This paper consists of several parts .In the part II we explore corner detectors and their properties .In the part III pose clustering algorithm and affine transform will be explained .In part IV, momentum algorithm as an approach for logo detection will be discussed .In part $\mathrm{V}$ conclusion of this article will come up.

\section{CORNER DETECTION}

A "corner "can be defined as the intersection of two edges. A corner can also be defined as a point that there are two dominant and different edge directions in a local neighborhood of the point. An interest point is a point in an image which has a well-defined position and can be robustly detected . This means that an interest point can be a corner but it can also be an isolated point of local intensity maximum or minimum, line endings, or a point on a curve where the curvature is locally maximal .In practice, most socalled corner detection methods detect interest points in general rather than corners in particular. As a consequence, if only corners are to be detected it is necessary to do a local analysis of detected interest points to determine which of these real corners are .Unfortunately, in the literature, "corner", "interest point "and "feature "are used somewhat interchangeable, which rather clouds the issue .Specifically, there are several blob detectors that can be referred to as "interest point operators", but which are sometimes erroneously referred to as "corner detectors"[11]Corner detectors are not usually very robust and often require expert supervision or large redundancies introduced to prevent the effect of individual errors from dominating the recognition task .The quality of a corner detector is often judged based on its ability to detect the same corner in multiple images, which are similar but not identical, for example having different lighting, translation, rotation and other transforms.

An alternative approach used frequently is based on a method proposed by Harris and Stephens, which in turn is an improvement of a method by Moravec.

Moravec algorithm [12]is one of the earliest corner detection algorithms and defines a corner to be a point with low self similarity. The algorithm tests each pixel in the image to see if a corner is present, by considering how similar a patch centered on the pixel is to nearby, largely overlapping patches . The similarity is measured by taking the sum of squared differences (SSD) between the two patches.A lower number indicated more similarity .One of the main problems with this operator is that it is not isotropic :if an edge is present that is not in the direction of the neighbors (horizontal, vertical and on the two diagonals), then it will be detected as an interest point.

Harris and Stephens [13], [14] improved upon Moravec's corner detector by considering the differential of the corner score with respect to direction directly, instead of using shifted patches .Limitations of Harris algorithm are : Computationally Demanding, Sensitivity to Noise, Anisotropic Response of Operator .For an example consider salt noise in the interior region of a black object. Any pixel affected by the salt noise will have a large gradient in all directions .That is, the salt noise is essentially an isolated pixel so has high intensity variation in all directions, and it is considered as a corner. The effect of noise is reduced by increasing the size of the Gaussian window as this causes more pixels to be considered when calculating the gradient, thus reducing the relative weight of any pixels affected by noise .Fig . 1 illustrated that the corners detected vary greatly with rotation that means Harris corner detector has anisotropic response.

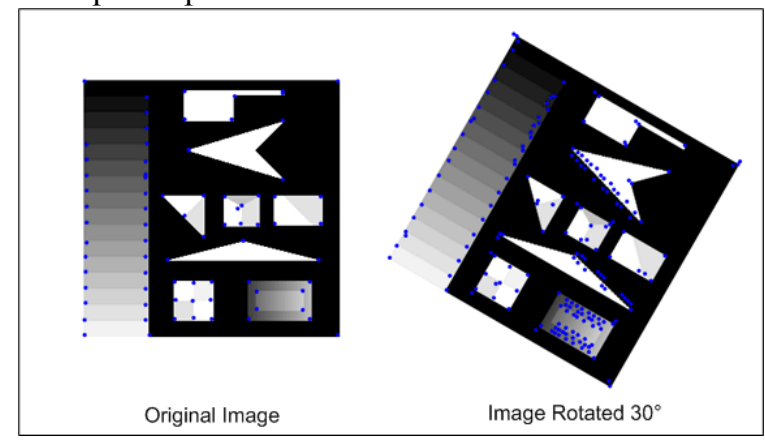

Fig .1 Rotational instability of Harris operator due to anisotropic response

With this method, unreal corners are increased by rotating image and are caused increasing computations. Then this operator is not desirable. Thus, we use the Fast corner detector.

In FAST (Features from Accelerated Segment Test) corner detection [15], the segment test criterion operates by considering a circle of sixteen pixels round the corner candidate $p$.The algorithm classifies $p$ as a corner if there exists a set of $n$ contiguous pixels in the circle which are all brighter than the intensity of the candidate pixel $I_{p}$ plus a threshold $T$, or all darker than $I_{p}-T$, as illustrated in Fig .2.

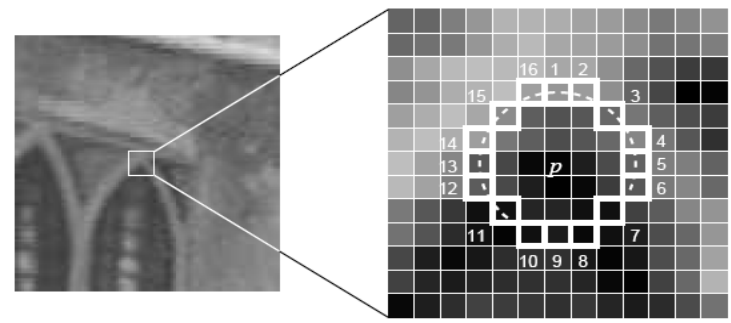

Fig .2 12 point segment test corner detection in an image patch. The highlighted squares are the pixels used in the corner detection. The pixel at $\mathrm{p}$ is the centre of a candidate corner. The arc is indicated by the dashed line passes through 12 contiguous pixels which are brighter than $\mathrm{p}$ by more than the threshold. 
$n$ was chosen to be twelve because it admits a high-speed test which can be used to exclude a very large number of non-corners :the test examines only the four pixels at 1, 5, 9 and 13 )the four compass directions. (If $p$ is a corner then at least three of these must all be brighter than $I_{p}+T$ or darker than $I_{p}-T$.If neither of these is the case, then $p$ cannot be a corner. The full segment test criterion can then be applied to the remaining candidates by examining all pixels in the circle. One sample of acting the Fast corner detector has been shown in Fig .3 .This detector in itself exhibits high performance, but there are several weaknesses .For example, the high-speed test does not generalize well for $n<12$, the choice and ordering of the fast test pixels contains implicit assumptions about the distribution of feature appearance, knowledge from the first 4 tests is discarded and multiple features are detected adjacent to one another.

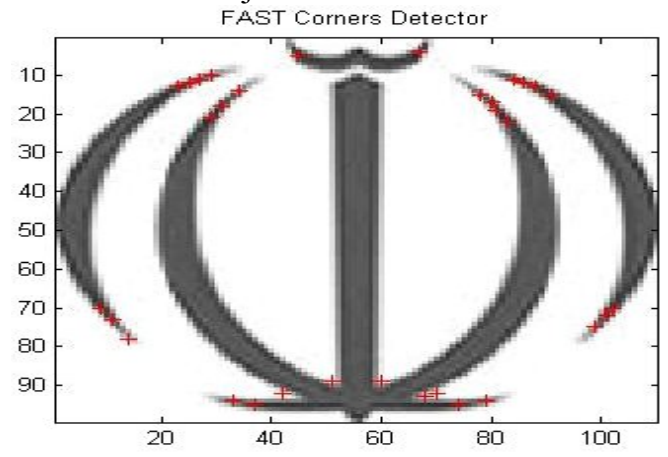

Fig.3 performance of FAST corner detection

\section{Logo Detection Using Pose Clustering}

In this section pose clustering algorithm [16] as a logo detection algorithm will be described .At first images shoul be converted to gray scale images . Then with using a corner detector, all corners in pictures should be detected as their feature points .As it has been explained in section II, we use FAST (Features from Accelerated Segment Test) corner detector [15] to extract corners .One of the output samples of this detector has been shown in Fig.4.

Pose clustering method works in this way that for every two points of feature points in training image and test image, affine transformation function is created. Then this transformation function will be applied to all the points of training image, and corresponded points to test image will be calculated .After that, the region corresponded to training image will be separated .Some examples have been shown in Fig. 5 .

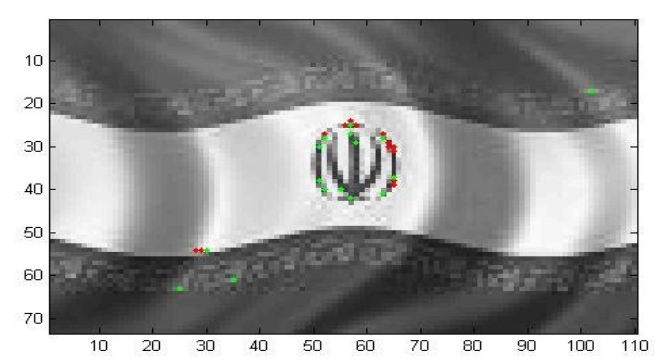

Fig.4 Corner detection using FAST algorithm

As it is obvious, with use of two points, we can calculate only linier transforms and we cannot distinguish skewing . For solving this problem we can use three points to be able calculating all affine transforms [17] .

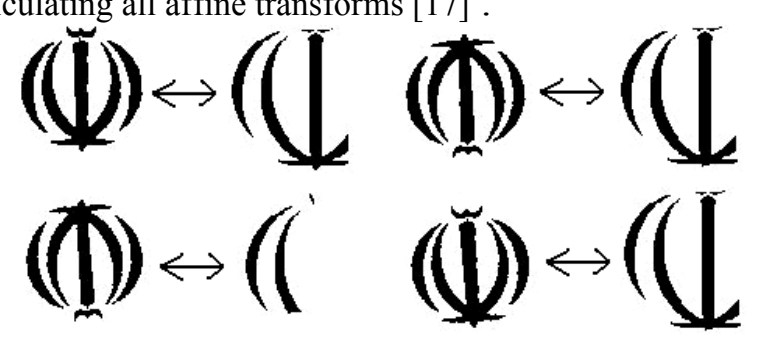

Fig.5 some samples for transformation and corresponded region in then test image

Afterwards to evaluate resemblance ratio between training image and corresponded region, we should use likeness criterion .This criterion can be selected from validation algorithms in image processing. One simple example for that can be ratio of black and white pixel that is corresponded to the same color, to all pixels in binary converted images .If two images match completely, this value will be 1 .Then if this value is nearer to 1 they are more similar .But because corner detector cannot extract the entire corners and extracted corners are not definitely accurate corners, so having some error is inevitable .Thus for more insurance we need to use another validation technique .We here use Euclidean distance from momentum $\varnothing_{1}, \varnothing_{2}, \varnothing_{3}$ and $\varnothing_{4}$ of training image to transformed image that has been produced from affine transform . We use this method for our secondary validation technique .Some performed results from this method have been exhibited in Fig. 6 and Fig. 7 .In Fig. 6 some transformed images that satisfy both resemblance criterion and momentum criterion, have been shown .In Fig.7 two false samples that do not satisfy our criterions have been demonstrated .

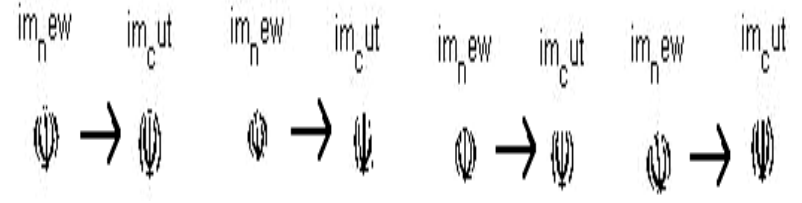

Fig.6 Some desirable transformation with a little tolerance that will be accepted 


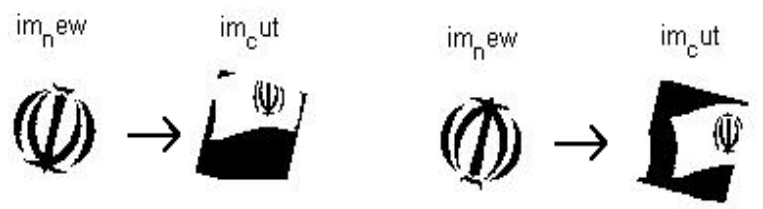

Fig.7 Some not desirable transformation that will be rejected

The significant properties of this approach are, being invariant to rotation, scaling, shifting, and skewing .With this method we can easily calculate the angle of rotation, the value of scaling and position of the logo .But two of the disadvantages of this approach are its time consuming characteristic and its requirement to much calculations .In this algorithm when number of corners increases, corresponded transformation functions increases rapidly.

At simulation between positive images in 71.7 percent of transformations, Iran logo has been existed and detected .In 28.3 percent of transformations, Iran logo has been existed and could not be detected .In 6.4 percent of transformations, Iran logo has not been existed and falsely detected as Iran logos .In 93.6 percent of transformations, Iran logo has not been existed and its output was correct .Also between negative images, in 8.1 percent of transformations, Iran logo has not been existed and falsely detected as Iran logos .In 91.9 percent of transformations, Iran logo has not been existed and its output was correct. Therefore in the bank of images in 82.6 percent of images that have Iran logo, its results were true and in 92 percent of images that have not Iran logo, its results were true.

\section{MOMENTUM METHOD FOR LOGO DETECTION}

In this section we will discuss about momentum as an independent method for logo detection, because momentums are invariant to rotation, scaling and shifting. So it looks like that they can be considered as a method for detecting logos . In this method after reading images, they will be converted to binary (black and white) images .Then after some preprocessing methods like Opening and Closing methods, it will be segmented into some connected component .After that momentum for every segment will be calculated .For training purpose, in training image, 100 affine transforms of it and their momentums will be calculated .Then we use average and standard deviation of results as training feature . For detecting purpose, momentums of every segment are checked to see if they are in range of average and standard deviation of training features or not .We here use Euclidean distance from momentum $\varnothing_{1}$ and $\varnothing_{2}$ of training image to test segment of our images. As it is clear, this method is a good detector in segments that their momentums are similar to the training momentums and we know that there is at least one same logo in image, and cannot work properly in images that do not include the logo, because it will suppose segment with similar momentum as our logo .For solving this problem we should use an accurate threshold that cannot obtain it easily and it depends on our banks. Therefore we should combine another simple method such as previous section to improve its results .One output sample of this method has been demonstrated in Fig.8.

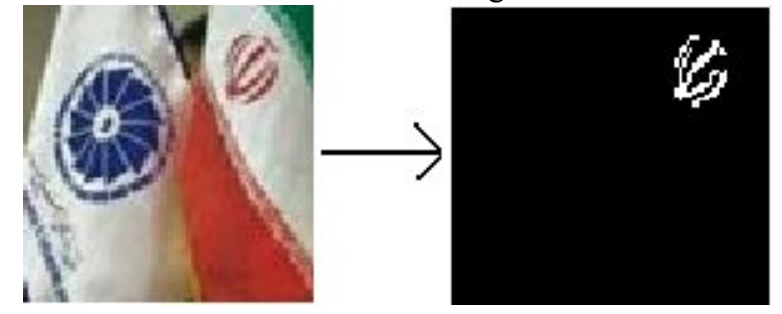

Fig.8 One sample of momentum result

At simulation in 70.7 percent of images containing Iran logo, they detected correctly, but only in 40 percent of images not containing Iran logo, the results were true .This method does not work properly for negative images but it works well for positive images in a high speed .

\section{CONCLUSIONS}

We use some methods for detecting logos in pictures . Pose clustering method combined with momentums (or at least one resemblance criterion) has high accuracy regards to mere momentum method and can detect skewing, too .But because the number of corners in a picture can be large, then the number of affine transformations may be very much and that makes it time consuming with a lot of calculations .And the second method (mere momentum method) has a high speed that works properly in same logo detection and for negative images it should be revised.

\section{REFERENCES}

[1] D .Doermann, "The Indexing and Retrieval of Document Images :A Survey", Computer Vision and Image Understanding, vol .70, no .3, pp .287-298.1998.

[2] D .Doermann, E .Rivlin, and I .Weiss .Applying algebraic and differential invariants for logo recognition .Machine Vision and Application:73-86, 1996.

[3] P .Suda, C .Bridoux, B .Kammerer, and G .aderlechner. Logo and word matching using a general approach to signal registration .In Proc .Int'l Conf .Document Analysis and Recognition, pages 61-65, 1997.

[4] J .Neumann, H .Samet, and A .Soffer .Integration of local and global shape analysis for logo classification .Pattern Recognition Letters, 1449-1457, 2002.

[5] K .Zyga, J .Schroeder, and R .Price .Logo recognition using retinal coding .In Proc .38th Asilomar Conf .Signals, Systems and Computers, volume 2, pages 1549-1553, 2004.

[6] G .Zhu, D .Doermann, "Automatic Document Logo Detection ",ICDAR 2007

[7] S .Seiden, M .Dillencourt, S .Irani, R .Borrey, and T . Murphy .Logo detection in document images .In Proc.Int'l Conf .Imaging Science, Sys., and Tech., pages 446-449, 1997.

[8] J.R .C'ozar, N .Guil, J.M .Gonz'alez-Linares and E.L .Zapata, " Video Cataloging Based on Robust Logotype Detection ", IEEE, CICY, 2006

[9] $\mathrm{K}$.Meisinger, $\mathrm{T}$.Troeger, $\mathrm{M}$.Zeller, and A .Kaup, "Automatic TV logo removal using statistical based logo detection and frequency selective inpainting", European Signal Processing Conference EUSIPCO Antalya, Sep .2005 
[10] M .Sonka, V .Hlavac, and R .Boyle, Image Processing, Analysis and Machine Vision .Chapman \& Hall Computing, London, 1993

[11] C .Damerval, S, Meignen, "Blob Detection With Wavelet Maxima Lines, "Signal Processing Letter IEEE, VOL.14, pp.29-42,2006

[12] X .Zhang, "Detection of moving corners in dynamic images", IEEE International Symposium, pp .36-41, 1994

[13] U .Orguner, F .Gustafsson, "Statistical Characteristics of Harris Corner Detector", IEEE Signal Processing, pp .571575,2007
[14] C.S .Kenney, M .Zuliani, B.S .Manjunath, "An axiomatic approach to corner detection "IEEE Computer Society Conference, Voll, pp.191-17, 2005

[15] S .Alkaabi, F .Deravi, "Candidate pruning for fast corner detection", IEEE Electronics Letters, Vol.40, pp.18-19, 2005

[16] S .Moss, E.R .Hancock, "Pose clustering with density estimation and structural constraints "IEEE Computer Society Conference, Vol.2, pp.90-91, 1999.

[17] J.S .Seo, J .Haitsma, T .Kalker, C.D .Yoo, "Affine transform resilient image fingerprinting "IEEE Signal Processing, Vol.3, pp.61-64, 2003 